\title{
DISEÑO DE UN SISTEMA DE SEGUIMIENTO SOLAR DE UN EJE PARA EL APROVECHAMIENTO DE LA ENERGIA SOLAR EN SISTEMAS FOTOVOLTAICOS
}

\author{
Responsable : : Ing. Manuel Benigno Gonzales Gámez \\ Miembros : : Ing. Alberto Enrique Cohaila Barrios \\ Ing. Edith Carmen Paredes Choque
}

\section{RESUMEN}

En este proyecto se hace el diseño y la implementación de un Sistema de Seguimiento Solar automatizado basado en el microcontrolador PIC 16F84. En la memoria del PIC esta embebido un programa de aplicación que envia una secuencia de pulsos digitales a un motor de posicionamiento angular. El motor esta acoplado a un engranaje de un eje rotacional que permite el desplazamiento horario de un panel fotovoltaico durante un tiempo de 6,7 minutos y un desplazamiento en giro antihorario de retorno a la posición inicial.

El desplazamiento angular de 1,2 grados permite orientarlo en la perpendicular de los rayos solares a cualquier hora del dia con un margen de error inferior a los 2,4 grados en total. Para la evaluación del seguidor solar de un eje, se tomarón 72 pasos en un tiempo de 8 horas de ángulo acimutal. El monitoreo de la radiación solar se realizó con una tarjeta de adquisición de datos USB interfaceado al computador. Los datos se almacenan en archivo de hoja Excel, para luego ser comparados con datos de un panel fotovoltaico estático.

\begin{abstract}
The present project includes the design and implementation of an automated System of Solar tracking based on the PIC 16F84 microcontrolador. The memory of the PIC has an application program that sends a sequence of digital pulses to an angular positioning motor. The motor this coupled to an engagement of a rotational axis which allows the time clock moment of a photovoltaic panel during 6,7 minutes and a counter clock movement in return to the initial position.
\end{abstract}

The angular displacement of 1,2 degrees allows to guide it to a perpendicular position of the sunbeam at any hour of the day, with a total margin error lower than 2,4 degrees. The evaluation of this one axis solar tracking has taken 72 steps in 8 hours of azimuthal angle. The solar has been registered using an acquisition date card USB interfaced to the computer. Data is stored in an Excel sheet file to be compared with the static photovoltaic panel.

\section{INTRODUCCIÓN}

La región Tacna está situada en la costa sur - occidental del Perú. Sus coordenadas geográficas se sitúan entre $16^{\circ}$ $58^{\prime}$ y $18^{\circ} 20^{\prime}$ de la latitud sur; y $69^{\circ} 28^{\prime}$ y $71^{\circ} 02^{\prime}$ de longitud oeste de Greenwich.

Esta región privilegiada en cuanto a la incidencia de radiación solar (del orden de los $5,5 \mathrm{KW} \mathrm{h} / \mathrm{m}^{2}$ promedio anual diario). La abundancia de radiación solar en la zona nos confiere un recurso valioso que promete un buen campo de desarrollo tecnológico energético.

En el presente trabajo de Investigación se construyó un sistema electrónico-mecánico que permite optimizar este proceso, orientando de manera automática paneles solares hacia la región de mayor incidencia de radiación e incrementando la eficiencia de éstos en la conversión de la radiación solar a energía eléctrica.

En el sistema desarrollado, se evalúan los niveles de radiación provenientes de dos puntos diferentes y actúan sobre un motor DC, para orientarse hacia el Sol.

El circuito puede usarse además, en otros sistemas que requieran la propiedad de heliotropismo, tales como: colectores solares y medidores de radiación directa.

El Centro de Energias Renovables cuentan con sistemas de aplicación de la energia solar, como son paneles fotovoltaicos, cocinas solares, secados solares etc. que permanecen en posición fija y durante cierto transcurso de tiempo utilizan la radiación solar a su máximo esplendor, lo cual no los hace eficientes debido a que sólo reciben la máxima radiación cuando esta en una orientación y hora determinadas. Los diversos sistemas diseñados para aprovechar la energia solar no cuentan con un sistema de monitoreo automático, asi como los paneles solares estáticos no aprovechan eficientemente la energia proveniente del Sol para una máxima eficiencia en el almacenamiento de energía en las batería. Se pretende diseñar e implementar un sistema de seguimiento del Sol de un eje en paneles fotovoltaicos.

\section{MATERIAL YMÉTODOS}

Para definir con precisión la posición del Sol en cada instante con respecto a un observador hipotético que se encontrase inmóvil en un plano horizontal, se utilizan dos coordenadas, llamadas altura solar $h$ y azimut solar $A$.

La altura es, sencillamente, el ángulo que forman los rayos solares sobre la superficie horizontal. A veces también se usa el llamado ángulo cenital o distancia cenital, que es el que forma el rayo con la vertical, es decir, el complemento de la altura (Fig. 1)

El azimut $A, 0$ ángulo azimutal, es el ángulo de giro del Sol medido sobre el plano horizontal mediante la proyección 
del rayo sobre dicho plano y tomando como origen el Sur. La Figura $\mathrm{N}^{\circ} 01$ ilustra la representación gráfica de la altura y el azimut. Por convenio, este último se considera negativo cuando el Sol está hacia el Este (por la mañana) y positivo cuando se sitúa hacia el Oeste (después del mediodia).

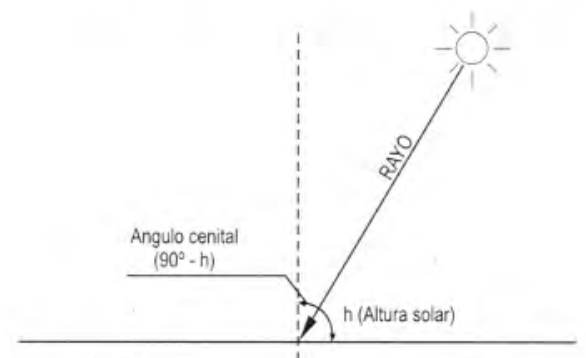

Figura $\mathrm{N}^{0}$ 01.- .Angulo cenital y altura solar

\section{1) Diseño y Construcción del Sistema de Seguimiento Solar de un Eje}

\section{Estructura del Seguidor Solar}

El diseño estructural del prototipo esta construido en material de aluminio. Esta debe soportar el peso del panel solar, el motor de posicionamiento angular, la tarjeta de control que hace posible el seguimiento del Sol y el mecanismo de dos rodamientos por cuyo eje atraviesa un tubo hueco, aprox. $60 \mathrm{~cm}$ de largo, que permite el libre movimiento del panel solar.

\section{Construcción del Seguidor Solar}

Un eje transversal hueco, suspendido en sus extremos por dos rodamientos standard de 1,6" de diámetro, se colocó encima del soporte rectangular alineado lo mas exactamente posible. Dos engranajes reductores de $2^{\prime \prime}$ y $2,5^{\prime \prime}$ de diámetro fue necesario acoplar entre el motor de posicionamiento angular y el eje transversal hueco. De esta manera se logra aumentar el torque del motor al doble aprox., consiguiendo mover asi elpanel solar.

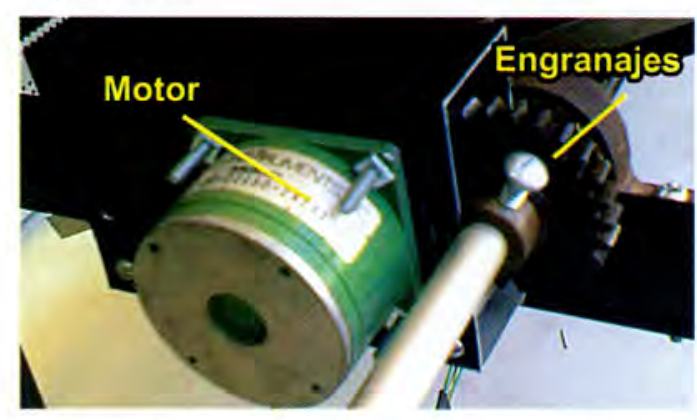

Figura $\mathrm{N}^{\circ}$ 02.- Engranajes del mecanismo de giro acoplado al motor paso a paso.

\section{2) Automatismo del Sistema de Seguimiento Solar de un Eje}

El principal elemento que hace posible el movimiento del panel solar es el motor de posicionamiento angular. Un circuito de control electrónico, cuyo cerebro es el microcontrolador 16F84, envia pulsos digitales, en código binario en secuencia de 4 bits, hacia el driver de corriente L293. El driver amplifica la baja corriente proveniente del PIC 16 F84 y satura (activa) a 4 transistores BD135, permitiendo fluir asi una corriente mayor aprox. 0,5 A entre colector $y$ emisor de cada transistor. La corriente de 0,5 A que fluye por cada bobina del motor de posicionamiento angular hace posible este movimiento. La alta corriente es necesaria para romper el torque estático y permitir el giro bidireccionalmente del panel solar.

\section{Elementos Principales de Control}

Se han considerado como elementos principales de control a elementos mecánicos y dispositivos electrónicos. Una tarjeta de control especialmente se ha diseñado con estos dispositivos:

- Motor de posicionamiento angular o motor paso a paso

- Microcontrolador PIC16F84

- Driver L293

\section{Diseño del Circuito de Control}

Este amplificador de corriente es necesario ya que entre el colector y emisor de Q1 Q4 (BD135) debe circular una alta corriente continua, más de $1 \mathrm{~A}$. Debido a que la corriente que entrega en la salida el opto transistor no es suficiente para excitar la base del transistor BD135 y lograr saturarlo. El driver permite amplificar la corriente a nivel suficiente como para poder circular corriente más de $1 \mathrm{~A}$ a través de Q1 Q4 cuando están saturados. Además que reduce la inserción de otros componentes como transistores y resistencias en la placa.

Las cuatro (4) señales que salen de Q1, Q2, Q3 y Q4 son señales paralelas de $12 \mathrm{Vdc}$ que permiten mover un motor paso a paso en sentido horario y antihorario. De esta forma se obtiene el seguimiento del panel solar hacia el Sol durante el dia y el retorno del panel solar, a la posición de origen, durante la noche.

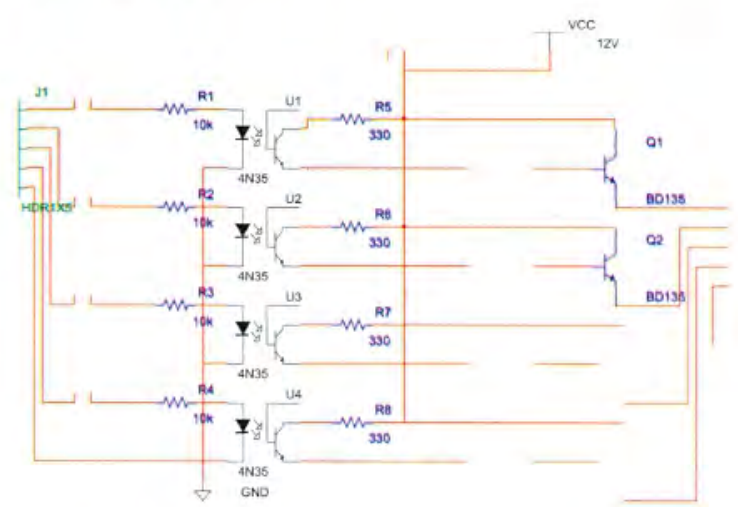

Figura $\mathrm{N}^{\circ}$ 03.- Diagrama circuital de la etapa de potencia

\section{Diseño del Programa}

El programa está hecho con lenguaje Assembler, lenguaje que entiende el microcontrolador PIC 16F84. Se muestra a continuación el código embebido que corre dentro del PIC16F84 que le da cierta inteligencia artificial al panel solar. 
:ESTE PROGRAMA PERMITE SEGUIR LA TRAYECTORIA DEL SOL EN LINEA RECTA :APROXIMADAMENTE CADA 7 MINUTOS AVANZA UN ANGULO DE 1.25 GRADOS.

\section{PIC 16F84A/ 4 MHz}

;Bits de configuración para el programador:

:CP(protección de la memoria de código): ..........................No

;PWRTE(activación del temporizador "Power-up") …..............No

;WDTE(activación del "perro guardián"):.................................. No

;FOSC1-FOSCO(tipo de oscilador):................................... Xtal

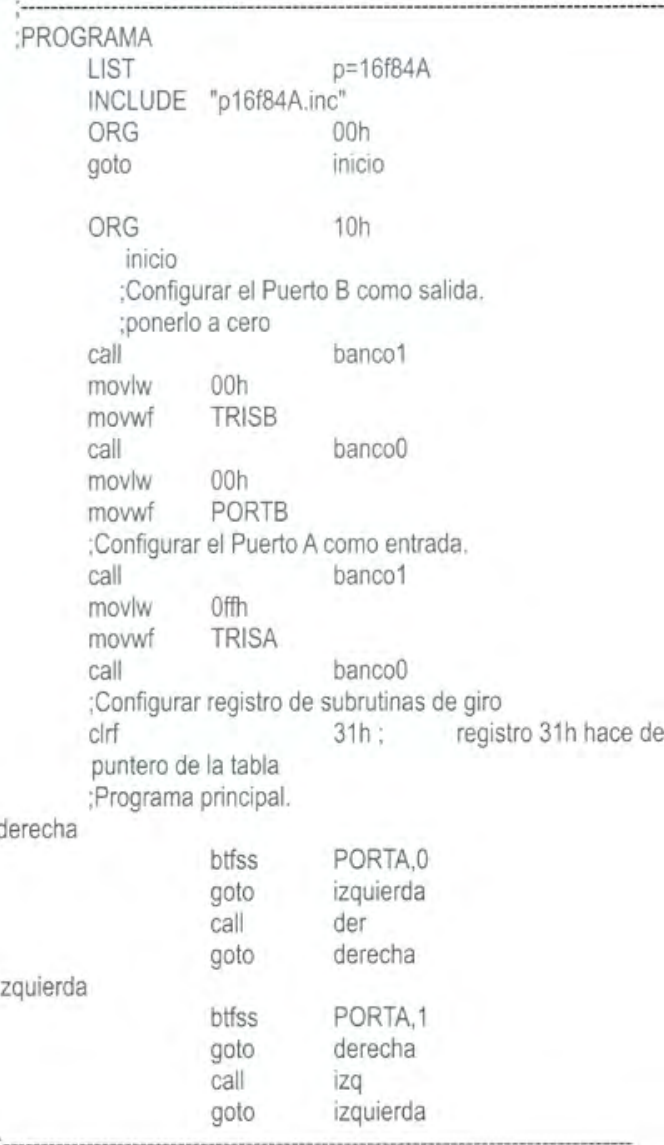

Subrutinas de giro a derecha e

izquierda del motor.

\section{Circuito de Control Basado en el PIC16F84}

Podemos observar las dos etapas principales para el funcionamiento del seguidor solar. EI PIC es un microcontrolador 16F84 en cuyo interior se encuentra un programa almacenado para un fin determinado, en este controlar y medir los tiempos de cada paso del motor. El circuito integrado L293B corresponde a la etapa de potencia.

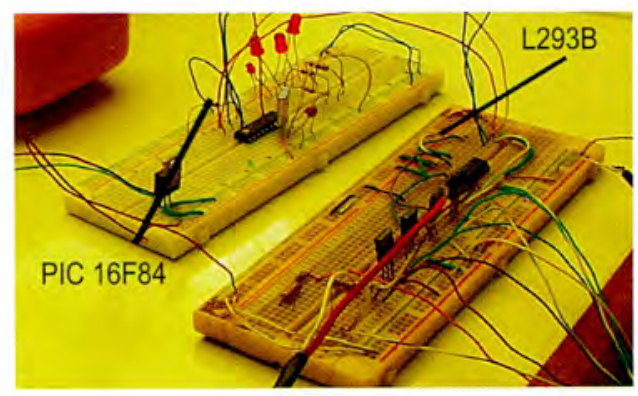

Figura No 04.- Etapa de control (PIC16F84)y Etapa de potencia (L293B).

\section{RESULTADOS}

Para la evaluación se utilizó un solarimetro y la tarjeta de adquisición de datos USB-6008 de National Instruments. Los datos fueron adquiridos en una computadora portátil y guardada en un archivo Excel para su posterior análisis.

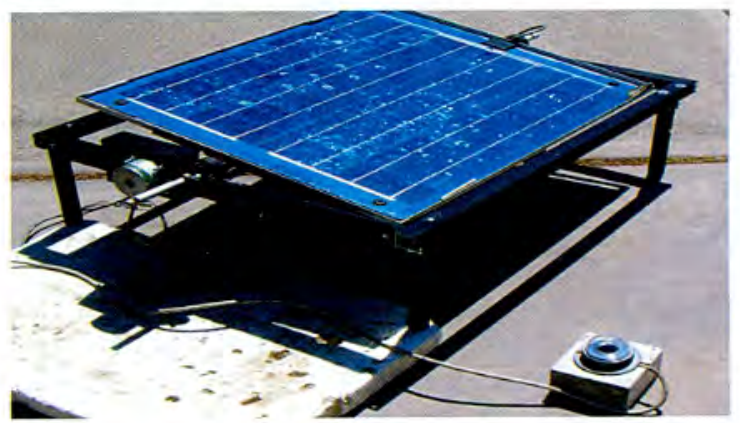

Figura $\mathrm{N}^{\circ}$ 05.- Panel solar con el solarimetro tomando datos

En la (Figura $N^{\circ}$ 5) se observa el modulo seguidor solar orientado en la dirección del desplazamiento del Sol. Para la evaluación del seguidor solar de un eje se tomarón 72 pasos en un tiempo de 8 horas de ángulo acimutal. El monitoreo de la radiación solar se realizó con una tarjeta de adquisición de datos USB interfaceado al computador. Los datos tomados se almacenan en archivo de hoja Excel, para luego ser comparados con datos de un panel fotovoltaico estático.

\section{1) Toma de Datos}

Cuadro $\mathrm{N}^{\circ}$ 01.- Datos de tensión y radiación obtenidos por la computadora.

\begin{tabular}{|c|c|c|c|c|}
\hline \multirow[t]{2}{*}{ MORA } & \multicolumn{2}{|c|}{ FOTOVOLTTALICO FWO } & \multicolumn{2}{|c|}{ 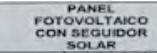 } \\
\hline & (v) & $\begin{array}{l}\text { Radicacibon } \\
\text { (Wim') }\end{array}$ & (v) & $\begin{array}{l}\text { Radidacibor } \\
\text { (Wtm) }\end{array}$ \\
\hline 10,00 & 8.4 & 570,093 & 10.5 & 122,81 \\
\hline 10,10 & 8.6 & 501,852 & 10.6 & 729,492 \\
\hline 10,20 & 9 & 619,38 & 10,0 & 742.250 \\
\hline 10,30 & 28 & 674,439 & 10,8 & 743.256 \\
\hline 10,40 & 10,4 & 715.728 & 11 & 787,02 \\
\hline 10.50 & 10.3 & 743.280 & & 791,43 \\
\hline 11.00 & 11.9 & 578,089 & 11,0 & 818,950 \\
\hline 11,10 & 11,50 & 791.43 & 11.80 & 812.078 \\
\hline 11,20 & 11,00 & 757.02 & 11,70 & 005,194 \\
\hline 1130. & 11,60 & 798,312 & 11,80 & a12,078 \\
\hline 11.40 & 12,00 & 825,84 & 11,00 & 818.958 \\
\hline 1150 & $11, .80$ & 812.076 & 11,100 & 012.078 \\
\hline 12.00 & 1130 & $7 m$ ses & 11,70 & 805,194 \\
\hline 12,10 & 11,00 & 757.02 & 11,40 & 784.548 \\
\hline 12.70 & 11,40 & 784,548 & 11,60 & 791,43 \\
\hline 12.30 & 11,50 & 791,43 & 11,80 & 812,076 \\
\hline 1240 & 11,00 & 757.02 & 11.30 & 777.606 \\
\hline 12,50 & 11,20 & 770,784 & 11.80 & 812.076 \\
\hline 13,00 & 10,10 & 780.138 & 12.00 & B25,8.4 \\
\hline 13.10 & 11,00 & 757.02 & 12,00 & 02504 \\
\hline 13.20 & 11.00 & 758.02 & 12,00 & 825,84 \\
\hline 13,30 & 10,60 & 729.492 & 11,00 & 757.07 \\
\hline 13.40 & 1020 & 701,964 & 11.50 & 701.43 \\
\hline 13.50 & 0,40 & 646.908 & 11,40 & 744,548 \\
\hline 14.00 & 8.00 & 674,436 & 11,30 & 777.006 \\
\hline 14,10 & 8,80 & $\cos 616$ & 10,50 & 722.61 \\
\hline 14,20 & 8,20 & 564,324 & 10.10 & 695,0022 \\
\hline 14.30 & 10,00 & 000.2 & $10+0$ & 715.728 \\
\hline 14.40 & 10.30 & 708,046 & 11,00 & 757,02 \\
\hline 14,50 & 10,30 & 708.826 & 10.50 & 722.61 \\
\hline 15.00 & 9.50 & 653,79 & 10.50 & 722,61 \\
\hline 15.10 & 7,80 & 523.032 & 9,500 & 600,79 \\
\hline 15.20 & 7,20 & 495,504 & 9.00 & 674,436 \\
\hline 15,30 & 0.40 & 440.448 & 9.50 & 653,79 \\
\hline 15.40 & 2.20 & 151.404 & 7,00 & $4 a 1,74$ \\
\hline
\end{tabular}

Fuente: Datos obtenidos el13/04/2007

\section{2) Evaluación del Seguidor Solar}

En la evaluación se observa claramente la eficiencia del seguidor solar en comparación con el panel fotovoltaico fijo. Nótese, en el Grafico 1, que durante las horas de mayor incidencia solar las curvas se aproximan. Y cuando la incidencia solar es menor las curvas se separan notoriamente. Observamos de esta forma la diferencia de un panel fotovoltaico fijo con el panel fotovoltaico con seguidor solar. 
GRAFICO No 01.- Curvas de tendencia de la tensión obtenida en dos paneles distintos.

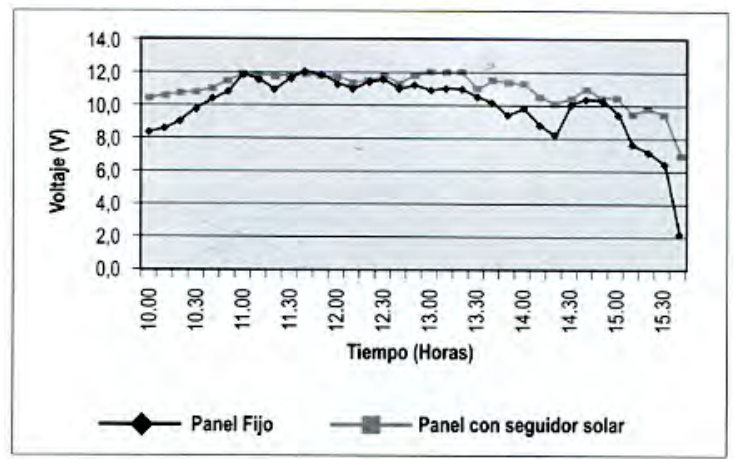

\section{DISCUSIÓN}

Este estudio estuvo enfocado en diseñar y construir un sistema de seguimiento solar de un eje a bajo costo usando tecnologia propia. El costo aproximado ascienda entre $100 \mathrm{y}$ 120 nuevos soles.

Durante la evaluación de dicho seguidor solar se pudo constatar que el viento puede llevar a un estado de inestabilidad al mecanismo de giro, esto no se tuvo en cuenta al momento del diseño y su construcción, pero deja abierto un tema a discutir en sistemas de seguimiento solar con control realimentado. La fuerza del viento (como variable perturbadora) debe ser eliminada. El seguidor solar de un eje de este proyecto presenta un modelo de control a lazo abierto. Un modelo de control a lazo cerrado elimina las perturbaciones.

Al ser un modelo experimental, el programa de usuario que corre dentro del microcontrolador puede ser modificado para mayor funcionalidad, como ser en estación de verano empezar el seguimiento del Sol desde tempranas horas (aprox. 7:30 am) hasta las 5.30 de la tarde en que se oculta el Sol.

Es de vital importancia proseguir con el desarrollo de la incipiente tecnologia de captación, acumulación y distribución de la energia solar, para conseguir las condiciones que la hagan definitivamente competitiva, a escala planetaria. Es por ello que se inicia este tipo de trabajo de investigación como aporte a esta nueva alternativa de captación de energía, usando seguidores solares diseñados y construidos en la Facultad de Ciencias de UNJBG.

\section{CONCLUSIONES}

1. Se logra realizar el diseño y la construcción del seguidor solar de un eje con elementos y componentes electrónicos accesibles en el mercado local.

2. Al demostrarse que los sistemas de seguidores solares son muy fiables mejoran los rendimientos energéticos y económicos de un consumidor final.

3. Se demostró que en un seguidor solar a pequeña escala, su panel fotovoltaico obtiene la máxima captación de energía durante todo el dia, de manera que el tiempo de vida de las baterias se alarga. Con lo cual el sistema aumenta entre un 15 y $20 \%$ los Kwh generados a través del campo fotovoltaico estático.

4. En contraste a un seguidor solar de doble eje, este logra aproximadamente del 35 al $40 \%$ de eficiencia en sistemas de gran potencia.

5. El consumo de corriente del circuito de control y los demás elementos es bajo, aproximadamente $0,65 \mathrm{~A}$, con una potencia de consumo de $3,3 \mathrm{~W}$.

6. El seguidor solar de un eje, con control de lazo abierto, debe mantenerse fuera de corrientes de aire para evitar su descalibracion a la hora de funcionamiento.

\section{BIBLIOGRAFÍA}

Centro de Energias Renovables (CER-UNI), Sociedad Alemana para la cooperación Técnica (GTZ).1991. Teoria y práctica del secador solar. Editora Hozlo SCRL. Lima.

Colegio de ingenieros del Perú. Curso Taller: "Usos prácticos de la Energía Solar" del 20 de junio al 15 de julio de 1983... Lima.

J. Guillermo Ugarte. Diseño y Evaluación de Experimentos para el Aprovechamiento de la Energia Solar. Tesis

H. Rodríguez y F. González. Manual de radiación solar en Colombia, Universidad Nacional de Colombia, Bogotá, (1992).

José M. Angulo Usategui. Microcontroladores Pic. 2da Edición Mc Graw-Hill/Interamericana de España, S.A.U. 2001.

Sayers I.L., Robson A.P. Principios de Microprocesadores 1ra. Edición, CECSAMéxico 1995. 